\title{
Adapting Conventional Tools to Analyse Ducted and Open Centre Tidal Stream Turbines
}

\author{
Steven Allsop* ${ }^{* \dagger}$, Christophe Peyrard ${ }^{\dagger}$, Pierre Bousseau ${ }^{\ddagger}$ and Philipp R. Thies ${ }^{\S}$ \\ *Industrial Doctoral Centre for Offshore Renewable Energy (IDCORE), \\ University of Edinburgh, Kings Buildings, Edinburgh EH93LJ, UK \\ E-mail: s.allsop@ed.ac.uk or steven-externe.allsop@edf.fr \\ †Electricité de France (EDF) R\&D LNHE, Research and Development, 6 Quai Watier, 78400 Chatou, France \\ ${ }^{\ddagger}$ Electricité de France (EDF) R\&D THEMIS, 7 boulevard Gaspard Monge 92120 Palaiseau, France \\ $\S$ College of Engineering, Renewable Energy Group, University of Exeter, Penryn Campus, TR109FE, UK
}

\begin{abstract}
This paper details a hydrodynamic model based on Blade Element Momentum Theory (BEMT) developed to assess 'conventional' 3-bladed tidal stream turbines (TSTs), adapted here to analyse an 'unconventional' case of a ducted and open centre device. Validations against a more detailed coupled Reynolds averaged computational fluid dynamics (RANS-BEM) model shows excellent agreement, of within $2 \%$ up to the peak power condition, with associated computational times in the order of a few minutes on a single core. The paper demonstrates the application of hydrodynamic forces into a structural analysis tool, in order to assess blade stress distributions of a generic hubless turbine. Incorporation of parameters such as non-uniform inflows and blade weight forces are investigated, with their effects on stress profiles presented. Key findings include: i) the adapted BEMT model replicates the majority of turbine performance characteristics estimated through previous CFD assessments; ii) the proposed model reduces the computational effort by several orders of magnitude compared to the reference coupled CFD, making it suitable for engineering assessments iii) blade stress distribution profiles are quantified, detailing concentration zones and cyclic values for use in fatigue analyses. This work forms part of a greater project aimed to develop a suite of analytical tools to perform engineering assessments of bi-directional ducted TSTs.
\end{abstract}

Keywords - tidal stream turbine, blade element, momentum theory, duct, open centre, structural analysis, blade stress.

\section{INTRODUCTION}

The tidal energy industry has witnessed significant breakthroughs in the last year, with the deployment of the first commercial scale arrays of tidal stream turbines (TSTs). This comes almost a decade after the installation of the MCT SeaGen 1.2 MW twin rotor device in the Strangford Loch, Northern Ireland in 2008, as one of the earliest landmark projects. The engineering challenges in designing reliable devices to withstand the harsh operating tidal environment is one of the main contributing factors for the limited rate of maturity seen in the industry. The MeyGen project Phase 1A has successfully installed and grid connected a 6 MW array in the Pentland Firth, Scotland, consisting of three Andritz Hydro Hammerfest and one Atlantis devices [1]. These are horizontal axis, three bladed turbines, similar in arrangement to modern wind turbines. A separate collaborative project between DCNS OpenHydro and EDF (Electricité de France) has seen the installation of two $500 \mathrm{~kW}$ devices in Paimpol
Bréhat, Northern France [2]. These are of a different turbine design, incorporating a rim generator housed in a bi-directional duct surrounding a high solidity, open centre rotor.

Significant developments in numerical modelling techniques have enabled highly detailed analyses of TST hydrodynamics, using complex computational fluid dynamics (CFD) simulations. Benefits include accurate representations of rotor performance, dynamic component loading, fluid/structure interactions, turbulent effects and wake formation. However, this comes at the price of high computational cost and long processing times. These models are therefore not ideal for use in engineering applications such as assessing structural performance of blades in various operating conditions.

Blade element momentum theory (BEMT) is a relatively simple but effective and well understood method of predicting turbine performance and rotor thrusts. Commonly used in the wind industry due to its fast running capabilities, the method has been applied more recently to tidal applications with commercial [3] and research-led [4] models. Despite its simplified approach, the low computational requirement makes this method suitable in making numerous engineering assessments. Current BEMT models have been extensively used to asses the performance and loads of three blades horizontal axis wind turbine. Extending these models to a bi-directional ducted, high solidity case with an open centre necessitate adaptations to the underlying principles of the model.

The aim of this paper is in twofold: 1) to present the development of a hydrodynamic model adapted from classical BEMT to analyse the blade loads on a ducted and open centre device, comparing against CFD RANS results for a generic ducted turbine proposed by the university of Oxford; 2) to demonstrate the use of the hydrodynamic forces in performing structural analyses on blades under various inflow conditions.

The remainder of the paper is structured into 5 key parts: the theory behind the Ducted BEMT model (II); the theory incorporated into the structural analysis tool (III); results and validation against a coupled CFD study (IV); structural analysis of blade stresses under various conditions (V); Conclusion and further work (VI). 


\section{HYDRODYNAMIC MODEL}

BEMT is a combination of blade element and momentum theories, and is well established methodology for analysing turbine forces, where many texts exist giving full equation derivations, such as [5], [3] and [6]. This study gives a condensed account of the principle theories, focussing primarily on the adaptations to the unconventional case. For further details on the development of the classical BEMT model that is used as a baseline here, the reader is referred to [7].

\section{A. Ducted Momentum Theory}

Linear momentum theory models the turbine as an infinitely thin actuator disc of zero friction, bounded by a control volume. Continuity and Bernoulli's equations can be used to relate the axial forces from the pressure change across the disc, to the change in axial momentum within the fluid caused by the presence of the disc restricting the flow.

Ducts are incorporated into TST designs to attempt to increase the mass flow rate through to the turbine, as well as providing other benefits such as aligning the flow and housing a rim generator. The hydrodynamic effects of the duct can be split into four components: i) the diffuser ratio (ratio of outlet area to throat area); ii) the flow separation within the diffuser; iii) the back pressure reduction at the exit and iv) the associated viscous losses [8]. A stream tube representation of the flow through a uni-directional duct is shown in Figure 1.

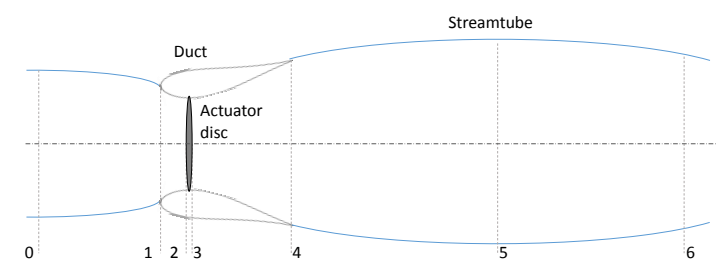

Fig. 1. Schematic of a uni-directional duct, highlighting geometrical parameters used in the empirical ducted momentum equations. Numerical values represent: 0 - far upstream, 1 - duct inlet, 2 - disc upstream, 3 - disc downstream, 4 - duct outlet, 5 - downstream, 6 - far downstream

An analytical model devised by [9] uses the pressure distribution and various duct geometries at various positions in order to assess the momentum changes within the stream tube. Specifically, the model is used to express efficiencies of duct inlet $\left(\eta_{02}\right)$ and diffuser efficiency $\left(\eta_{34}\right)$, as well as the base pressure coefficient $\left(C_{p, b}\right)$, which can be resolved using CFD in order to calculate the various pressures and velocities.

$$
\begin{aligned}
\eta_{02} & =\frac{p_{2}-p_{0}}{1 / 2\left(U_{0}^{2}-U_{2}^{2}\right)} \\
\eta_{34} & =\frac{p_{4}-p_{3}}{\frac{1}{2} \rho U_{3}^{2}\left(1-\frac{A_{3}^{2}}{A_{4}^{2}}\right)} \\
C_{p, b} & =\frac{p_{0}-p_{4}}{\frac{1}{2} \rho U_{0}^{2}}
\end{aligned}
$$

Where denoted values are at each location along the streamtube: $U_{n}$ is fluid velocity $\left(\mathrm{ms}^{-1}\right), p_{n}$ is the pressure $(\mathrm{MPa})$ and $A_{n}$ is the area $\left(\mathrm{m}^{2}\right)$.
RANS simulations incorporating an actuator disc representation of the rotor are made on unidirectional ducts [10], based on NACA0015 aerofoil shapes [11]. Many different geometries are tested, varying: inlet contraction ratio $\left(A_{1} / A_{3}\right)$; the diffuser expansion ratio $\left(A_{4} / A_{3}\right)$; inner and outer diffuser surface angles $\left(\theta_{\text {in }}\right.$ and $\left.\theta_{\text {out }}\right)$, as defined in Figure 2.

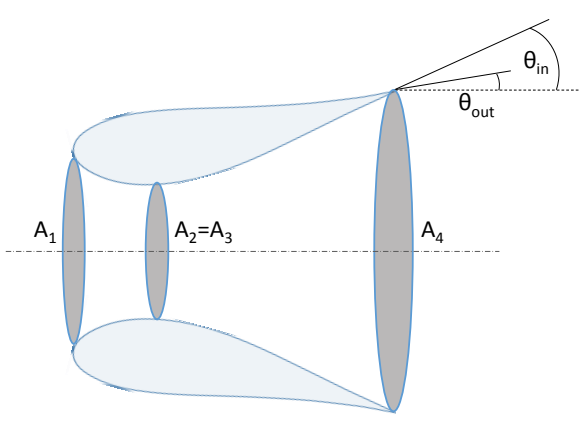

Fig. 2. Schematic of a uni-directional duct, indicating geometrical parameters used in the empirical expression development

From the results of these studies, empirical approximations for each of the analytical expressions is defined:

$$
\begin{aligned}
\eta_{02} & \approx 1.0 \\
\eta_{34} & =a_{1}+b_{1} \frac{A_{3}}{A_{4}}+c_{1} \theta_{\text {in }}+d_{1} \frac{A_{1}}{A_{2}} \\
C_{p, b} & =a_{2}+b_{2} \frac{A_{3}}{A_{4}}+c_{2} \theta_{\text {out }}+\ldots \\
& \ldots\left(d_{2}+e_{2} \theta_{\text {out }}\right) C_{T i}+f_{2} C_{T i}^{2}
\end{aligned}
$$

The inlet efficiency was found to be within $5 \%$ of unity for all cases tested, therefore assuming a value of 1.0 is thought to have negligible effects on the overall rotor forces. $C_{T i}$ is the thrust coefficient calculated using thrust from blade element theory (equation 12). All coefficients were determined using a least squares optimisation function, detailed in Table I.

TABLE I

COEFFICIENTS FOR EMPIRICAL EXPRESSIONS OF DIFFUSER EFFICIENCY $\left(\eta_{34}\right)$ AND BASE PRESSURE COEFFICIENT $\left(C_{p, b}\right)$, TAKEN FROM [10]

\begin{tabular}{llllll}
\hline a1 & b1 & c1 & d1 & & \\
0.8867 & 0.5212 & -0.0108 & -0.1313 & & \\
\hline a2 & b2 & c2 & d2 & e2 & f2 \\
0.2701 & -0.333 & 0.0269 & 0.1068 & -0.0152 & -0.1275 \\
\hline
\end{tabular}

The pressure change through the diffuser can be derived from the continuity equations, such that the coefficient of pressure between positions 3 and 4 can be written:

$$
C_{p, 34}=\frac{p_{4}-p_{3}}{\frac{1}{2} \rho U_{3}^{2}}=\eta_{34}\left(1-\frac{A_{3}^{2}}{A_{4}^{2}}\right)
$$

Leading to an expression for the axial induction factor as follows, where wake swirl is neglected:

$$
1-a=\sqrt{\frac{\eta_{02}-C_{T i}+C_{p, b}}{\eta_{02}-C_{p, 34}}}
$$


Where $a$ is the axial induction factor. This empirical / analytical model is validated in the same study, against power and thrust results from additional duct geometries, with reasonable agreement [10].

\section{B. Rotational Momentum}

Rotational momentum is gained by the flow in the wake which can be equated to the torque transmitted to the rotor. As this is a function of tangential velocity, the disc is split into a number of annular rings, where torque applied to each ring is expressed as:

$$
d Q=4 \pi \rho a^{\prime} \Omega U_{0}(1-a) r^{3} d r
$$

Where $\rho$ is the fluid density $\left(\mathrm{kgm}^{-3}\right), a^{\prime}=\omega / 2 \Omega$ is the tangential induction factor, dQ is the element torque $(\mathrm{N} \mathrm{m}), \omega$ the angular velocity of the wake $\left(\mathrm{rad} \mathrm{s}^{-1}\right)$ and $\Omega$ the angular velocity of the turbine $\left(\operatorname{rad~s}{ }^{-1}\right)$.

\section{Blade Element Theory}

Blade element theory splits the blades into a number of discrete hydrofoil sections, which are analysed two dimensionally, neglecting the spanwise (three dimensional) interactions. At each 2D element, there are associated axial and tangential components of flow velocity, with the inflow angle $(\phi)$ located between.

The aerodynamic forces of lift and drag on the blade elements act parallel and perpendicular to this inflow angle, determined using typical aerofoil expressions [3]:

$$
\begin{aligned}
d L & =\frac{1}{2} \rho C_{L} W^{2} c d r \\
d D & =\frac{1}{2} \rho C_{D} W^{2} c d r
\end{aligned}
$$

Where $\mathrm{W}$ is the resultant fluid velocity $\left(\mathrm{m} \mathrm{s}^{-1}\right)$ and $\mathrm{c}$ the blade chord $(\mathrm{m})$. Coefficients of lift $\left(C_{L}\right)$ and drag $\left(C_{D}\right)$ vary as a function of angle of attack $(\alpha)$, which can be determined from the inflow angle, and the geometrical twist down the blade length $(\beta)$. The forces axial thrust and torque can then be resolved such that:

$$
\begin{aligned}
d F_{a x} & =\frac{1}{2} \rho W^{2} B c\left(C_{L} \cos (\phi)+C_{D} \sin (\phi)\right) d r \\
d Q & =\frac{1}{2} \rho W^{2} B c\left(C_{L} \sin (\phi)-C_{D} \cos (\phi)\right) r d r
\end{aligned}
$$

Where $\mathrm{B}$ the number of blades and $\phi$ the inflow angle $\left(^{\circ}\right)$.

\section{Numerical Implementation}

Ducted BEMT is a combination of both theories, where it is assumed that the change in momentum is solely accountable from the aerodynamic forces on the blade elements. The equations are solved iteratively using a code written in programming language Python. Thrust and torque expressions from the blade element and momentum theories can be combined into a single factor $(g)$, as per similar solvers [4], where a minimisation function can be applied:

$$
\begin{aligned}
g & =\left[\eta_{02}-C_{T i}+C_{p b}-\left(\eta_{02}-C_{p 34}\right)(1-a)^{2}\right]^{4} \ldots \\
& +\left[4 a^{\prime} \sin (\phi) \cos (\phi)-\sigma_{r}\left(1+a^{\prime}\right)\left(C_{L} \sin (\phi) \ldots\right.\right. \\
& \left.\left.-C_{D} \cos (\phi)\right)\right]^{4}
\end{aligned}
$$

Where the local blade solidity $\sigma_{r}=B c / 2 \pi r$. Each side of the equation is raised to the power 4 , to avoid convergence on an incorrect solution and found to enhance numerical stability.

The minimisation function within the SciPy library is comprised of numerous optimisation algorithms, which are selected based on problem parameters [12]. For the present case, Sequential Least Squares Programming (SLSQP) is used as the best compromise between computational time, convergence and operational constraints. An iteration limit is set to 1,000 , and a tolerance value of $g$ set to $1.0 \mathrm{E}-10$. Boundary limits of induction factors are also applied in order to ensure values stay within a reasonable range, where $a^{\prime} \leq 0.5$ and $-0.8 \leq a \leq 0.8$.

\section{E. Power and Thrust}

After convergence is achieved, coefficients of power $\left(C_{P}\right)$ and thrust $\left(C_{T}\right)$ give non-dimensional indications of rotor performance, often quoted against tip speed ratio $(T S R)$, and defined as:

$$
\begin{aligned}
C_{T} & =\frac{\sum_{r_{h u b}}^{R} d F_{a x}}{\frac{1}{2} \rho A U_{0}^{2}} \\
C_{P} & =\frac{\sum_{r_{h u b}}^{R} d Q \Omega}{\frac{1}{2} \rho A U_{0}^{3}} \\
T S R & =\frac{\Omega R}{U_{0}}
\end{aligned}
$$

Where $A=\pi R_{d u c t}^{2}$ is the area of the disc $\left(\mathrm{m}^{2}\right)$. In addition, the thrust coefficient at the local elements can be calculated:

$$
C_{T, l o c}=\frac{d T}{\frac{1}{2} \rho d A U_{2}^{2}}
$$

Where $d A=2 \pi r d r$ is the area of the annular ring $\left(\mathrm{m}^{2}\right)$.

\section{F. Correction Factors}

Under highly loaded conditions, thrust forces are under predicted by the BEMT model due to limitations in the fundamentals of the stream tube assumptions. Therefore a semi-empirical correction factor, based on a variation [13] of the Glauert parabola [5] is imposed, as detailed in [7].

A reduction in hydrodynamic efficiency occurs at the blade tips and root due to radial flow, but is neglected by BEMT due to the 2D flow assumptions. A correction factor $(\mathrm{F})$ can be introduced as devised by Glauert [5], which proves effective in conventional devices [4]. However, as a result of the blade tips being connected by an outer ring housed inside the duct, there is a restriction on the formation of the tip vortices. CFD studies have reported very small changes in axial velocity at these locations [14], and therefore the tip losses are set to unity. Three dimensional flow is thought to occur around the hub geometry, but is likely to be highly influenced by complex wake mixing with flow through the open centre. A hub loss factor could be devised, however would require input from detailed blade resolved CFD simulations. The hub loss in this study is neglected due to a limitation of this data. 


\section{G. Non-uniform Inflow}

The Ducted BEMT model can also solve for non-uniform inflow profiles, which are steady and 'frozen', where elemental axial velocity is determined as a function of its position in the water column. This velocity is calculated for each azimuthal angle, and used to calculate the corresponding elemental forces. An azimuthal stepping function is applied to attain the cyclic forces during each rotation. As an example, a shear profile is applied to estimate the effects of sea bed friction. The inflow velocity is be expressed as a $1 / 7^{\text {th }}$ power law, where the elemental velocity can be written:

$$
U_{0}=U_{h u b}\left(\frac{z}{z_{h u b}}\right)^{\frac{1}{7}}
$$

Where $U_{0}$ and $U_{h u b}$ are the elemental and hub height velocities $\left(\mathrm{ms}^{-1}\right)$; and $z$ and $z_{h u b}$ are the elemental and hub height above the seabed respectively $(\mathrm{m})$.

\section{Structural AnAlysis}

The axial and tangential forces acting on the blades can be translated into stresses in order to make structural assessments. These can be used to give indications on survivability of blades under extreme loading, as well as fatigue damage from cyclic loading associated to non-uniform inflows. Here, we consider a generic device with a bi-directional duct and no central hub, where the blades are considered completely open at the rotor centre.

\section{A. Bending Moment and Shear Force}

Initially, forces from the global coordinate system are translated to local coordinate systems at each element, using the blade twist:

$$
\begin{aligned}
& \text { Flapwise }: F_{z}=F_{\text {tan }} \cos (\beta)-F_{a x} \sin (\beta) \\
& \text { Edgewise }: F_{y}=F_{t a n} \sin (\beta)+F_{a x} \cos (\beta)
\end{aligned}
$$

The blade can be modelled as a cantilever beam, fixed at one end representing the connection to the outer rim on the turbine, and free at the opposite end representing the open centre, as shown in Figure 3. Shear forces and bending moments can then be calculated at various positions along the blade.

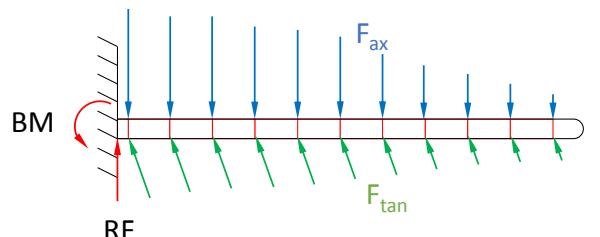

Fig. 3. Cantilever beam representation of a turbine blade, showing axial and tangential force distributions as well as highlighting root bending moment $(\mathrm{BM})$ and resultant force $(\mathrm{RF})$

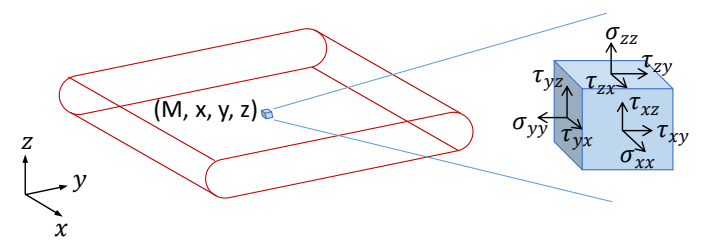

Fig. 4. Diagram of stress vectors for a single generic blade element

\section{B. Stress Tensor Matrix}

Stress vectors for a single generic blade element can be defined using structural mechanics fundamentals [15], as shown in Figure 4.

These vectors can be combined into a stress tensor matrix, expressed as:

$$
[\sigma]=\left[\begin{array}{lll}
\sigma_{x x} & \tau_{x y} & \tau_{x z} \\
\tau_{y x} & \sigma_{y y} & \tau_{y z} \\
\tau_{z x} & \tau_{z y} & \sigma_{z z}
\end{array}\right]
$$

Normal and shear stresses can be defined as below, where some stress components are assumed negligible for this case, and therefore are set to zero:

$$
\begin{aligned}
\sigma_{x x} & =\frac{F_{x}}{A}-\frac{M_{z} z}{I_{y}}+\frac{M_{y} y}{I_{z}} \\
\sigma_{y y} & =\sigma_{z z}=0 \\
\tau_{x y} & =S F \frac{F_{y}}{A} \\
\tau_{x z} & =S F \frac{F_{z}}{A} \\
\tau_{y x} & =\tau_{y z}=\tau_{z x}=\tau_{z y}=0
\end{aligned}
$$

The normal stress along the blade axis $\left(\sigma_{x x}\right)$ is a combination of bending stresses and direct stress (as a function of blade weight forces). $A$ is the cross sectional area of the blade element $\left(\mathrm{m}^{2}\right), M_{y}$ and $M_{z}$ are the bending moments $(\mathrm{Nm})$ in each direction; $\mathrm{z}$ and $\mathrm{y}$ are the perpendicular distances from the neutral axis to the surface (m) and $I_{y}$ and $I_{z}$ are second moment of area about the neutral axis $\left(\mathrm{m}^{4}\right)$. Shear stresses are a function of coefficient of shear $\mathrm{SF}=1.5$, specific to a rectangular cross section, with the maximum shear located at the neutral axis.

In order to combine the stress tensor into a singular parameter for comparison, the Von Mises stress can be defined by:

$$
\sigma_{v m}=\sqrt{\sigma_{x x}^{2}+3\left(\tau_{x y}^{2}+\tau_{x z}^{2}+\tau_{y z}^{2}\right)}
$$

\section{HYDRODYNAMIC MODEL VALIDATION}

The present Ducted BEMT model is applied to a generic turbine developed by the University of Oxford, where a hydrodynamic model has been used to assess the performance using a coupled RANS-BEM model. Full details of the model are published [16], with further information available in the $\mathrm{PhD}$ thesis of the same lead author [17]. 


\section{A. Input Definitions}

Overall turbine geometry is defined in Figure 5. Diffuser inlet and outlet surface angles are, however, not detailed, which are required inputs into the Ducted BEMT model. As these are not easily defined for bi-directional ducts, a comparative study was performed to estimate appropriate values, calibrating against a blade resolved CFD study. Results gave optimal values of $\theta_{\text {in }}=10^{\circ}$ and $\theta_{\text {out }}=30^{\circ}$.
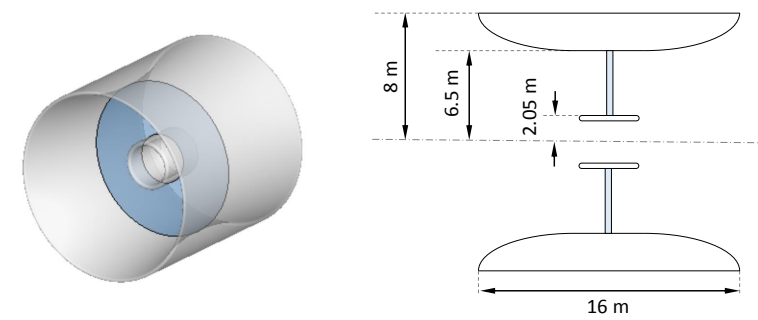

Fig. 5. Generic turbine overall geometry (reproduced from [16])

Radial distributions of blade twist $(\beta)$ and local solidity $\left(\sigma_{r}=B c / 2 \pi r\right)$ are as defined in Table II.

TABLE II

NORMALISED RADIAL DISTRIBUTION OF TURBINE GEOMETRIES

\begin{tabular}{lll}
\hline$r / R(-)$ & $\beta\left(^{\circ}\right)$ & $\sigma_{r}(-)$ \\
\hline 0.3 & 29.7 & 0.420 \\
0.4 & 25.6 & 0.305 \\
0.5 & 20.8 & 0.220 \\
0.6 & 17.2 & 0.163 \\
0.7 & 14.2 & 0.124 \\
0.8 & 12.0 & 0.100 \\
0.9 & 10.3 & 0.083 \\
1.0 & 8.4 & 0.070 \\
\hline
\end{tabular}

A single flow condition is considered, based on a uniform inflow with no bottom friction, at a constant velocity of $\mathrm{ms}^{-1}$. This has a corresponding chord based Reynolds number of approximately $1.0 \mathrm{E}+06$. The TSR is varied by changing only the disc rotational velocity.

\section{B. Aerofoil Coefficients}

Blades are made up of Risø-A1-24 aerofoil sections, with lift and drag coefficients as shown in Figure 6, taken from wind tunnel data at a Reynolds number of 1.6E+06 [18]

For extreme angles of attack, coefficients of the last quoted value are extrapolated, such that when $\alpha>35^{\circ}$, values at $35^{\circ}$ are used and $\alpha<-5^{\circ}$, values at $-5^{\circ}$ are used. This is in order to be consistent with the validation methodology, as there were no occasions where converged angles went outside the range $-5^{\circ}<\alpha<35^{\circ}$ in the test case for a bare turbine.

\section{Results}

Figure 7 shows the non-dimensionalised coefficients of power $\left(C_{P}\right)$ and thrust $\left(C_{T}\right)$ variation with TSR, which follow

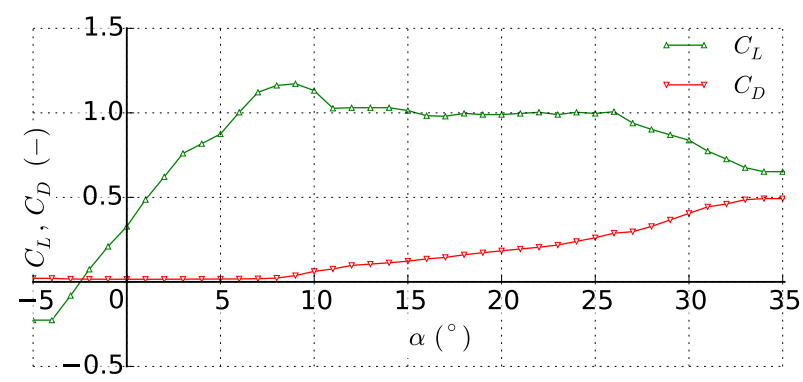

Fig. 6. Coefficients of lift and drag against angle of attack for a Riso-A1-24 aerofoil from wind tunnel tests, $\mathrm{Re}=1.6 \mathrm{E}+06[18]$

an increasing trend up to a peak power condition, before decreasing at a lower rate. Here it can be seen that the results from the Ducted BEMT model are in excellent agreement with the coupled RANS-BEM model (within $2 \%$ ), up to the optimal TSR $=3$. Beyond the peak, a disparity is seen, increasing with TSR, where the present study predicts up to $25 \%$ higher power, and $10 \%$ higher thrust at TSR $=5$.

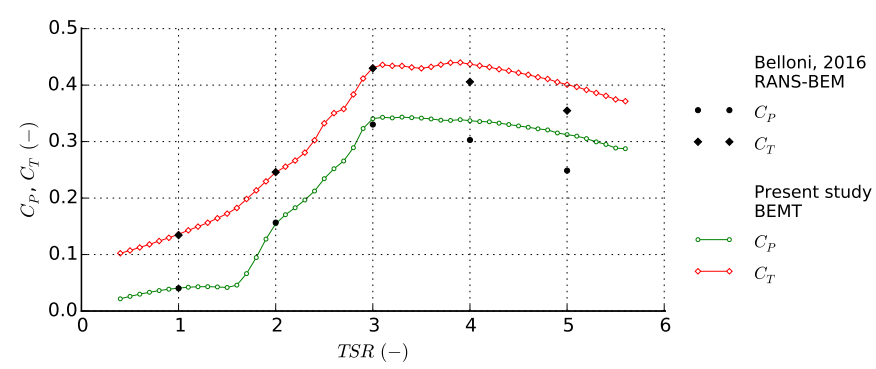

Fig. 7. Rotor coefficients of power and thrust variation with TSR for a full scale open centre and ducted turbine, comparing present ducted BEMT with RANS-BEM results [16]

Figure 8 shows radial distributions of: normalised velocity at the disc $\left(U_{d} / U_{0}\right)$, angle of attack $(\alpha)$ and local element coefficient of thrust $\left(C_{\text {Tloc }}\right)$. It can be seen that in general, the trends from the current study match the coupled CFD results well, confirming that the model is performing similarly at individual element scale as well as for the entire rotor. Slight differences are apparent in the thrust and velocity predictions at TSRs 4-5, which are responsible for the disparity seen in the overall rotor results. Here, we can identify that the differences are localised at low $r / R$ values, at elements situated around the open centre hub.

\section{Discussion}

Rotor power and thrust predictions are very similar for both models at TSR $<4$, including at the peak operating condition. Both methods implement the blade element theory, based on identical geometrical parameters, lift and drag coefficients and correction factors. Therefore, this aspect is not considered an area for comparison, moreover the difference is only attributed to the treatment of fluid momentum. The suggestion that can be taken from the results of this study is that there are very similar momentum changes calculated within the empirically modified momentum theory compared to that computed with a 


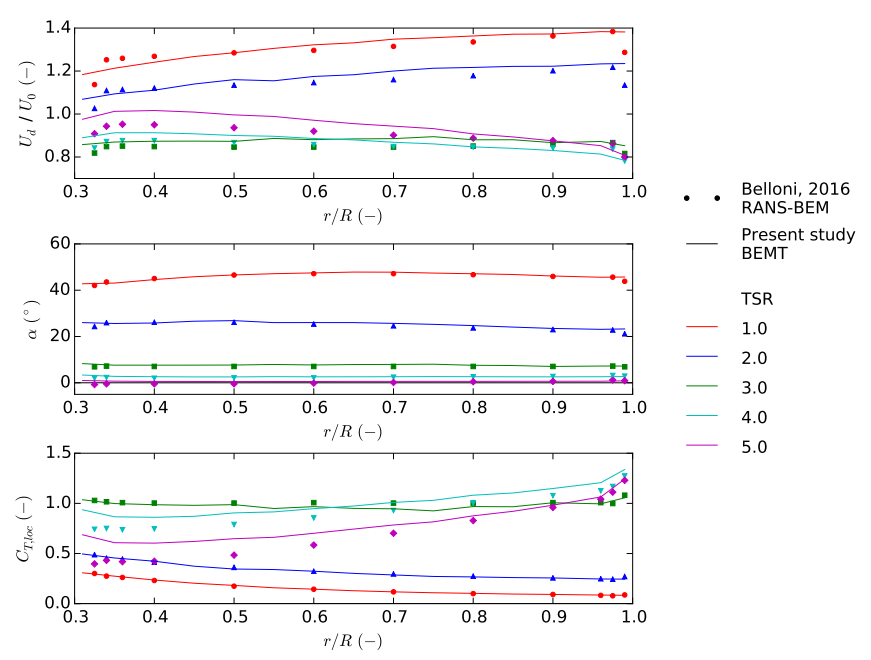

Fig. 8. Variations of a) normalised flow velocity (top), b) angle of attack (middle) and c) local elemental thrust coefficient (bottom) with normalised radius, for various tip speed ratios, comparing Ducted BEMT (lines) with RANS-BEM (solid markers) [16]

RANS CFD model. Further analysis shows that this is also true all along the rotor radius, at a local element level. Although this has positive indications, there is a limited number of data points for comparison, and therefore trends cannot be fully verified.

Higher power and thrust forces are predicted by the Ducted BEMT model at higher TSR $\geq 4$. This is thought to be associated to more complex flow characteristics, which are better captured by the more detailed CFD. Further detailed analysis shows the difference is due to higher local velocities predicted at the hub. Flow interactions around the hub and through the open centre are thought to reduce the hydrodynamic efficiency of blade elements around this region. It is also noted that angles of attack are very low, and in the region that aerofoil lift and drag curves gradients are greatest, suggesting a high sensitivity. The occurrence of these discrepancies is at TSR outside of the optimal range, and therefore thought to only be influential if the device employs over speed control in order to limit the blade forces in fast tidal flows.

Although a correction factor is applied to highly loaded conditions in the model, it is only activated when the axial induction factor goes beyond the transition into the highly loaded regime. As a $<0.4$ at all conditions, this factor is never applied, and therefore does not influence the results.

In order to remain consistent with the validation study methodology, lift and drag coefficients are kept constant outside a range of angles of attack $-5^{\circ}<\alpha<35^{\circ}$. At TSR $=1$ the angles of attack are outside this range at all radial locations. A post stall treatment could be therefore applied, such as the Viterna extrapolation function [19], used in NREL software [20] to improve the accuracy of the aerodynamic coefficients.

\section{E. Computational Requirements}

The RANS-BEM study was reportedly performed on a 16 node computer cluster, with 8 cores per node. Steady computations of the ducted case were completed in 8 hours using 4 cores, equivalent to 32 core hours for each of the 5 TSR points [17]. By contrast, the present Ducted BEMT study were performed using a laptop running an Intel Core $e^{T M} i 5$ $2.9 \mathrm{GHz}$ dual core processor with $8 \mathrm{~GB}$ RAM. Simulations were completed within 3 minutes, generating all 60 points on the power and thrust curves, equivalent to $\sim 6$ core minutes.

Computational time from two separate studies is difficult to directly compare, due to dependencies on factors such as the computer used, processor type, number of partitions, clock time etc. However, differences of several orders of magnitude seen in this study is indicative of substantial computational cost savings. This highlights an advantage of using the current model in the application of performing engineering assessments such as fatigue damage or multiple design iterations.

\section{F. Modelling Limitations}

Both models rely on the blade element theory, where each individual section is taken as an independent aerofoil, discounting any spanwise flow. Various pre-processing tools can be applied to correct for various physical occurrences that are not accounted, hence reducing the impact of modelling assumptions. One technique is to apply correction factors to account for the delayed stall of a rotating blade, such as the Du-Selig and Eggers functions [21]. However, these have only been seen applied to conventional devices, and so may require adaptation when applied to different configurations.

The effect of the duct on the change in fluid momentum are devised from CFD studies on uni-directional duct geometries. In order to apply these empirical expressions, the diffuser inlet and outlet angles must be defined. As these are not given in the data source, estimations are made based on a calibration study using results from blade resolved CFD. There are inevitably errors associated to this approach, due to the differences in geometry, as well as in the different numerical method used to generate the calibration data.

Hub losses are neglected in the model, which causes over predictions in blade forces comparing with CFD results, particularly at high TSRs. This is also seen to affect the spanwise forces, which will have implications on the stress calculations for blade sections around the hub.

The inflow is considered perpendicular to the rotor plane, as well as inviscid and steady, therefore not accounting for dynamic effects such as turbulence or inertia. Quasi-static simulations using a time stepping function imposing different frozen inflows could be performed, however is beyond the scope of this study.

\section{Blade Stress Results}

The rotor distributions of force can be used to calculate the blade stresses, where an analytical tool has been developed based on cantilever beam theory. As a demonstration of the model capability, the elemental forces output by the Ducted BEMT for a single TSR are fed into the structural tool. A generic blade structure is assumed, based on a generic bi-directional and hub-less turbine, where the blade stress 
distribution across the whole disc is presented. Effects of non uniform inflow and of ratio of buoyancy to weight are additionally tested.

\section{A. Input Parameters}

A generic turbine blade is defined, to demonstrate the application of the model to real TSTs. A simplified cross section is analysed, based on a flat plate aerofoil section with rounded edges with a uniform solid interior structure, as detailed in Figure 9. This is a simplifying assumption, where in reality a blade will be constructed from composite materials with various outer surface treatments and internal structures. A $\mathrm{TSR}=2$ is selected to give a good compromise between force magnitude and visibility of the effects from the different parameters tested. The blade stresses are output as a function of radial and azimuthal position, which can be combined into polar plots representing the turbine frontal area. Azimuthal angles increase clockwise, from $0^{\circ}$ at the westward location.

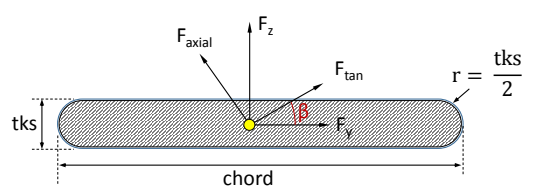

Fig. 9. Generic turbine blade element dimensions for structural analysis

\section{B. Uniform Inflow}

Figure 10 shows the distribution of blade stresses around the rotor radius, for all azimuthal angles in one turbine rotation. It can be seen there is no azimuthal variation, as the inflow velocity is constant everywhere across the disc. From this figure, it is possible to locate stress concentration zones along the blades. Here it can be seen that the peak stress is calculated at $8.84 \mathrm{MPa}$, and occurs approximately halfway along the blade length.

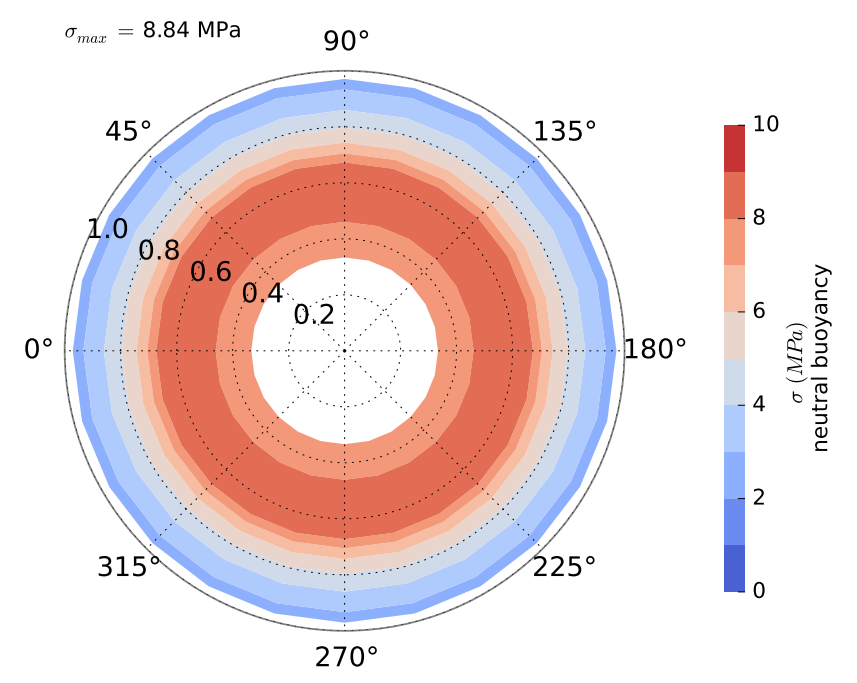

Fig. 10. Polar plot of Von Mises Stress distribution around the rotor radius, for different azimuthal angles, using a uniform inflow velocity profile at 2 $\mathrm{ms}^{-1}$, for a $\mathrm{TSR}=2.0$

\section{Non-uniform Inflow}

A non-uniform inflow profile to approximate the vertical shear from sea bed friction is applied, based on a $1 / 7^{\text {th }}$ power law at $2 \mathrm{~ms}^{-1}$ hub height. It can be seen that this causes a variation with azimuth, as the blade elements pass through different velocities at different locations in the water column. The distribution is symmetrical, as the inflow profile is $2 \mathrm{D}$, with no transverse variations. Peak stress has increased to 9.48 $\mathrm{MPa},(7.2 \%$ higher than the uniform case), located approximately halfway down the blade. A peak stress concentration zone occurs at azimuths around $90^{\circ}$, at the top dead central position.

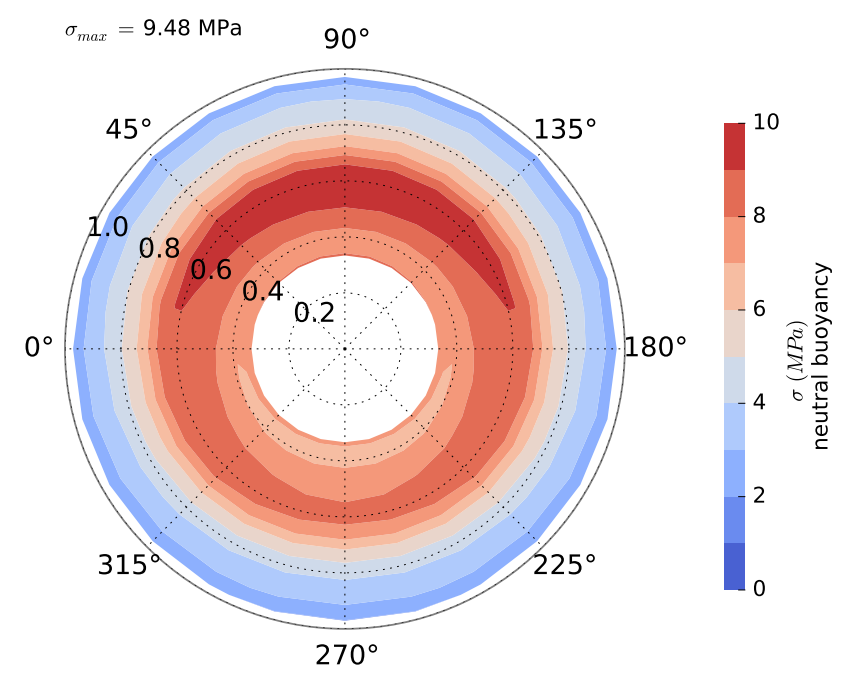

Fig. 11. Polar plot of Von Mises Stress distribution around the rotor radius, for different azimuthal angles, using a non-uniform inflow velocity profile of a $1 / 7^{\text {th }}$ power law at $2 \mathrm{~ms}^{-1}$ hub height, for a TSR $=2.0$

\section{Buoyancy and Weight Forces}

Stresses are affected by the weight volumetric force as well as pressure forces acting on the surface. A simplifying assumption is made whereby an effective local weight of $20 \%$ of the real weight is considered along the length of the blade to account for pressure forces. This corresponds to approximately $2 \mathrm{Kn}$, based on a similar TST [22], which is distributed along the length of the blade, proportional to the element volume. Peak stress is calculated at $9.52 \mathrm{MPa}(0.4 \%$ greater than for the neutral buoyancy case). Again, a stress concentration zone is identified towards the blade centre, however here there is a shift in position by approximately $20^{\circ}$ clockwise.

\section{E. Discussion}

Numerous inflow velocities and TSRs are tested, with similar stress profiles observed: minimum values located at the blade root, before increasing to a maximum approximately halfway along the blade length, before finally reducing towards the tip. This is because the stresses are a function of both forces and geometry. At the root, bending moments are highest, however there are smaller stresses due to the larger sized the elements. At the tips, on the other hand, 


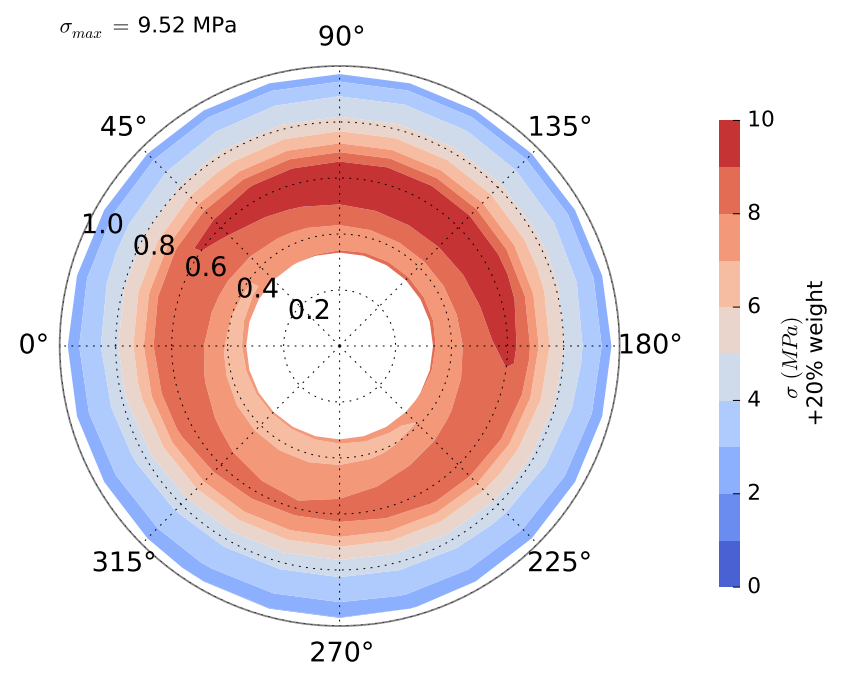

Fig. 12. Polar plot of Von Mises Stress distribution around the rotor radius, for different azimuthal angles, with a $20 \%$ weight force applied, using a nonuniform inflow velocity profile of a $1 / 7^{\text {th }}$ power law at $2 \mathrm{~ms}^{-1}$ hub height, for a $\mathrm{TSR}=2.0$

elements have small forces, but are much smaller in size, so their associated stresses are higher. The blade centres are identified as the highest stress areas. If, in other operational cases, stresses go beyond acceptable limits, the model can be used to identify areas that can be improved to effectively increase blade survivability.

Changing the uniform inflow to a shear profile causes an azimuthal variation in stress. The maximum stress concentration zone is again located towards the midpoint along the blades, however is seen at the top dead centre turbine position. This is due to the higher velocities seen at azimuths which correlate to where the blade is at the highest point in the water column. If blade fatigue assessments are required, the model can output the cyclic stresses at any element in the domain. Using this case as an example, it can be seen that stresses at the central blade element vary by $3 \mathrm{MPa}$ during one rotation, indicating that stresses can vary by $30 \%$ resulting purely from the addition of a simple shear profile.

Incorporating a simple representation of the blade weight forces does not have a significant effect on the maximum stress seen. The varying pressure distribution on the blade surfaces as a result of the depth variations could be incorporated to better represent the turbine physical effects, however is not thought to be a significant influence on global peak stresses. A shift in the stress concentration zone is seen to occur, by approximately $20^{\circ}$ clockwise. As the turbine rotates, the direction in which the weight force is applied changes. When the blade is vertical (at positions $90^{\circ}$ and $270^{\circ}$ ), the force acts vertically down the blade, with an associated direct stress. When the blades are in a horizontal position (at positions $0^{\circ}$ and $180^{\circ}$ ), the force acts in the tangential direction, and combined with those from the hydrodynamic model causing rotor torque. As the turbine rotates clockwise, when the weight forces act in the same direction as the tangential hydrodynamic force (between $\left.91^{\circ}-269^{\circ}\right)$, they are summed, resulting in higher stresses. The contrary occurs when the forces act in the direction opposing turbine rotation (between $271^{\circ}-89^{\circ}$ ).

The computational time increases almost linearly with each azimuthal angle added to be analysed, due to the time dependency being mostly related to the iterative loop for convergence in the hydrodynamic model. Regardless, calculations remain very short, in the order of minutes using a single processor.

\section{CONCLUSION}

A hydrodynamic model based on BEMT for assessing 'conventional' 3-bladed TSTs is adapted for application to an 'unconventional' case of a high solidity, ducted and open centre device. The Ducted BEMT incorporates an empirical formulation of momentum changes through a duct, devised from CFD studies and based on an analytical framework. The model is validated against a coupled RANS-BEM study, with good agreement seen in power and thrust predictions of within $2 \%$ for low TSRs, including the peak power condition. Higher rotational velocities show differences of up to $25 \%$ in power, and $10 \%$ in thrust, due to higher flow velocities predicted around the hub with the Ducted BEMT model. This is thought to be due to limitations in capturing complex 3D flow, which are thought more significant at higher TSRs, as well as around the hub geometry and the open centre. Computations are completed on a dual core processor within a few minutes, indicating significant cost savings compared to the reference coupled CFD study.

A demonstration is made of how these quickly generated hydrodynamic forces from Ducted BEMT can be used in engineering applications. A structural analysis tool is developed, designed to translate individual element forces into blade stresses. This can be subsequently used to calculate the peak stress, as well as to identify stress concentration zones, results of which help to inform survivability assessments. Examples are presented of how non-uniform inflow profiles and blade weight forces can be incorporated. Corresponding results suggest influences on the magnitude of the peak stresses, as well as the stress distribution around the rotor. These results will enable calculation of cyclic stresses under different operating conditions, to be ultimately fed into a fatigue assessment.

\section{FURTHER WORK}

A rain-flow counting function is currently being incorporated into the model, in order to translate the cyclic stresses from different inflow conditions into blade fatigue damage.

Dynamic effects such as waves are thought to have a high impact on the fatigue life of the blades, however are currently neglected from the BEMT model. These could be accounted through the addition of a dynamic wake model for the momentum and a dynamic stall model for the blade theory, and is currently being investigated.

Improvements to the hydrodynamic model are also to be made through further comparison to blade resolved CFD studies, which will give indications on the influence from 
blade element assumptions. Further verification of the model accuracy will also be made with measurements from physical experiments. Losses through reductions in hydrodynamic efficiency at the central hub can be assessed using the detailed CFD, which could form a basis of a new loss correction factor to feed into the model.

\section{ACKNOWLEDGEMENTS}

This research is carried out as part of the Industrial Doctoral centre for Offshore Renewable Energy (IDCORE), funded by the Energy Technology partnership and the RCUK Energy programme (Grant number EP/J500847/1), in collaboration with EDF R\&D.

\section{REFERENCES}

[1] MeyGen, "MeyGen Phase 1A Project Update Spring 2016," 2016. [Online]. Available: http://www.meygen.com/wp-content/uploads/ Meygen-Newsletter-201602.pdf

[2] DCNS OpenHydro, "OpenHydro Paimpol-Brehat Project Update," 2016. [Online]. Available: http://www.openhydro.com/OpenHydro/ media/Documents/NewsPDFs/30-May-2016.pdf

[3] DNV GL Garrad Hassan, "Tidal Bladed Theory Manual," Tech. Rep., 2012.

[4] I. Masters, J. C. Chapman, J. a. C. Orme, and M. R. Willis, "Modelling high axial induction flows in tidal stream turbines with a corrected blade element model," 3rd International Conference on Ocean Energy, pp. 15,2010 .

[5] T. Burton, N. Jenkins, D. Sharpe, and E. Bossanyi, Wind Energy Handbook, 2nd ed. John Wiley \& Sons Ltd, 2011.

6] P. J. Moriarty and a. C. Hansen, "AeroDyn Theory Manual," Tech. Rep. 2005. [Online]. Available: http://www.nrel.gov/docs/fy05osti/36881.pdf

[7] S. Allsop, C. Peyrard, P. R. Thies, E. Boulougouris, and G. P. Harrison, "A validated BEM model to analyse hydrodynamic loading on tidal stream turbine blades," in 3rd Asian Wave and Tidal Energy Conference, 24-28 October 2016, vol. 2, Singapore, 2016.

[8] G. J. W. van Bussel, "The science of making more torque from wind: Diffuser experiments and theory revisited," Journal of Physics: Conference Series, vol. 75, no. 012010, 2007.

[9] C. J. Lawn, "Optimization of the power output from ducted turbines," Proceedings of the Institution of Mechanical Engineers, Part A: Journal of Power and Energy, vol. 217, no. August 2002, pp. 107-117, 2003.

[10] M. Shives and C. Crawford, "Developing an empirical model for ducted tidal turbine performance using numerical simulation results," Proceedings of the Institution of Mechanical Engineers, Part A: Journal of Power and Energy, vol. 226, no. 1, pp. 112-125, 2011. [Online]. Available: http://pia.sagepub.com/content/226/1/112.abstract

[11] I. H. Abbott and A. E. Von Doenhoff, Theory of Wing Sections (Including a Summary of Airfoil Data). Langley: Dover Publications Inc, New York, 1959. [Online]. Available: http://www.journals.uchicago. edu/doi/10.1086/470266

[12] SciPy Community, "SciPy reference guide," 2016. [Online]. Available: https://docs.scipy.org/doc/scipy-0.18.1/reference/index.html

[13] M. L. Buhl, "A New Empirical Relationship between Thrust Coefficient and Induction Factor for the Turbulent Windmill State," National Renewable Energy Laboratory, Tech. Rep., 2005.

[14] C. F. Fleming and R. H. J. Willden, "Analysis of bi-directional ducted tidal turbine performance," International Journal of Marine Energy, vol. 16, pp. 162-173, 2016.

[15] K. D. Hjelmstad, Fundamentals of Structural Mechanics, 2nd ed Springer, 2005.

[16] C. Belloni, R. H. J. Willden, and G. Houlsby, "An investigation of ducted and open-centre tidal turbines employing CFD-embedded BEM," Renewable Energy, 2016.

[17] C. Belloni, "Hydrodynamics of Ducted and Open-Centre Tidal Turbines (PhD Thesis)," Ph.D. dissertation, The University of Oxford, 2013.

[18] P. Fuglsang, K. Dahl, and I. Antoniou, "Wind tunnel tests of the Ris $\varnothing$ A1-18, Ris $\emptyset$-A1-21 and Ris $\emptyset$-A1-24 airfoils,” Ris $\emptyset$ National Laboratory, Roskilde, Denmark, Tech. Rep., 1999.
[19] L. a. Viterna and D. C. Janetzke, "Theoretical and experimental power from large horizontal-axis wind turbines," US Department of Energy, Conservation and Renewable Energy Wind Energy Technology Division, Tech. Rep., 1982.

[20] S. A. Ning, "AirfoilPrep documentation, Technical Report NREL/TP5000-58817 (release 0.1.0)," National Renewable Energy Laboratory, Tech. Rep. June, 2013.

[21] S.-P. Brenton, F. N. Coton, and M. Geir, "A Study on Different Stall Delay Models Using a Prescribed Wake Vortex Scheme and NREL Phase VI Experiment," in EWTEC 2007, 2007.

[22] G. S. Bir, M. J. Lawson, and Y. Li, "Structural design of a horizontalaxis tidal current turbine composite blade," Tech. Rep. October, 2011. 
